\title{
Uso de métodos cuantitativos y cualitativos en el estudio del consumo de bienes culturales en sectores populares de Mexicali, B. C.
}

\author{
Luz María Ortega Villa*
}

\begin{abstract}
Resumen. En este trabajo se presenta de manera sucinta la metodología con la que se ha abordado el consumo de bienes culturales en sectores populares de Mexicali, que incluye tres fases: aplicación de una encuesta (técnica cuantitativa), elaboración de una tipología a partir de análisis multivariado (minería de datos), y realización de entrevistas a casos-tipo seleccionados (técnica cualitativa), las cuales son analizadas desde una perspectiva multidimensional cuyas categorías recuperan los aportes de la mercadotecnia respecto de la elaboración de visiones del consumo (Phillips, Olson y Baumgartner, 1995), la puesta en marcha de estrategias de resolución de conflictos (Kwai-Choi y Collins, 2000) en la toma de decisiones de consumo de bienes culturales, y las contribuciones de la sociología (Thompson, 1990; Bourdieu, 1984) para la identificación de aspectos reproductivos del consumo relacionados sobre todo con el capital educativo y el origen social, así como para el registro de estrategias de evaluación simbólica de los bienes culturales legitimados.
\end{abstract}

Palabras clave: consumo cultural, bienes culturales, metodología, tipología, cultura, frontera

\begin{abstract}
In this work, a brief of the methodology used in the study of cultural goods consumption among popular sector of Mexicali is presented. It includes three phases: a survey (quantitative method), a typology established from a multivariate analysis (data mining), and interviews with selected type-cases (qualitative method) all of which are analyzed from a multidimensional perspective whose categories retrieve marketing findings such as consumption visions (Phillips, Olson and Baumgartner, 1995) and conflict resolution strategies (Kwai-Choi and Collins, 2000) applied in the process of decision-making. It also draws from sociology (Thompson, 1990; Bourdieu, 1984) in order to identify reproductive aspects of consumption related to educative capital and social origin, and to deal with symbolic evaluation strategies of legitimate cultural goods.

Key words: cultural consumption, cultural goods, methodology, typology, culture, border
\end{abstract}

\footnotetext{
* Facultad de Ciencias Humanas, Universidad Autónoma de Baja California. Correos electrónicos: lucyo@uabc.mx, lucyov9@hotmail.com
} 


\section{Introducción}

En el campo de las ciencias sociales, el consumo ha sido estudiado tradicionalmente desde perspectivas que apuntan hacia las prácticas manifiestas involucradas en aquél, esto es, la adquisición de productos o servicios por parte de los consumidores. Qué es lo que se compra, cuándo y dónde, han sido las principales interrogantes, pero poco se ha estudiado el porqué de las elecciones. En particular, los enfoques de la economía toman como punto de partida la racionalidad del consumidor como un agente libre de tomar decisiones con base en el máximo beneficio; los aspectos subjetivos e individuales de las elecciones -que en ocasiones parten del supuesto de la libertad del consumidor- son abordados por la psicología, mientras que desde las ciencias de la comunicación se ha llegado a poner énfasis en la capacidad de los medios masivos para orientar las decisiones de consumo, a la vez que se ha declarado que existen mediaciones que intervienen en ellas. La "sociedad de consumo" como objeto de estudio recibió especial atención en los últimos cincuenta años, sobre todo a partir de los postulados de la Escuela de Frankfurt sobre el papel de las industrias culturales en la conformación de la cultura y la sociedad contemporáneas.

Por su parte, los estudios culturales, en tanto herederos de una tradición sociológica que incorpora aportes de la antropología, han abordado sobre todo lo relativo al consumo/recepción de obras artísticas, la decodificación de textos literarios y la significación de mensajes. Junto con el texto clave de Mary Douglas y Baron Isherwood (1979), El mundo de los bienes. Una antropología del consumo, la obra de Pierre Bourdieu (1984) titulada La distinción hizo evidente el vacío que había existido en la sociología respecto del estudio del consumo de productos culturales y los factores que en dicho proceso inciden. No obstante, todavía es poco lo que se ha estudiado sobre los procesos de toma de decisiones en la elección de los bienes culturales.

En nuestra región, la obra El consumo cultural en América Latina (Sunkel, 1999) es un libro clave pues reúne trabajos y perspectivas de las diversas latitudes; y en México merece un lugar especial el trabajo de Néstor García Canclini (1993), El consumo cultural en México, al pre- 
sentar argumentos para una propuesta teórica interdisciplinaria y exponer resultados de estudios hechos, sobre todo, en el centro del país. Si bien en ambas obras los trabajos incluidos abordan el consumo cultural con base en métodos tanto cuantitativos como cualitativos (asistencia a espacios culturales, recepción de medios masivos, experiencias en museos, recepción de obras artísticas, etc.), nuevamente parten del momento en el que el consumidor/receptor realiza el consumo, que García Canclini (1993: 24) define como "el conjunto de procesos socioculturales de apropiación y usos de los productos", y no se preguntan sobre cómo fue que llegó a tomar la decisión de consumir.

En contrapartida, el consumo de productos y servicios no específicos del campo de la producción cultural (Bourdieu, 1993), como son electrodomésticos, alimentos, automóviles, vacaciones, etc., es continuamente estudiado por las agencias encargadas de colocar dichos bienes y servicios en el mercado, así como de monitorear las ventas de los mismos, para lo cual recurren también al establecimiento de perfiles de los consumidores y a estudios sobre la toma de decisiones que se lleva a cabo tanto en el ámbito familiar como en distintos grupos de edad. Enlistar los trabajos publicados requeriría más páginas de las que este trabajo tiene, de ahí que baste con señalar que tan sólo a través del servicio EBSCO-Host, se encontraron más de 4 mil fichas relativas a dichos temas, y son apenas las que están disponibles en revistas académicas, a lo cual habría que agregar los estudios que realizan las agencias de mercadotecnia o las empresas mismas para conocer las preferencias de los consumidores y las razones de ellas, trabajos que se mantienen como reportes internos que no se dan a conocer al público.

Con base en lo anterior, y sobre todo debido a la carencia de información respecto de ese tema en el contexto de nuestra entidad, a mediados del año 2003 se dio inicio al proyecto de investigación titulado "Consumo de bienes culturales en sectores populares de la ciudad de Mexicali, B.C." que, financiado por la Universidad Autónoma de Baja California (UABC) y con apoyo del Instituto de Cultura de Baja California, se propuso identificar las características del consumo de bienes culturales en los sectores de población mencionados, cuyos resultados fueron publicados en la obra Donde empieza la carne asada. Consumo de bienes culturales en sectores populares de Mexicali (Ortega y 
Ortega, 2005). Posteriormente, como continuación del trabajo, en 2005 nuevamente la UABC aprobó y otorgó recursos para la realización del estudio denominado "Consumo de bienes culturales en sectores populares: decisiones y elecciones", cuyo objetivo fue identificar elementos (educativos, familiares, contextuales, etc.) que inciden en la decisión de consumo de bienes culturales, y que incluyó la elaboración de una tipología. ${ }^{1}$ De dichos trabajos se desprende el proyecto de tesis doctoral titulado "El consumo de bienes culturales en sectores populares: un enfoque multidimensional", que recupera los resultados de ambos proyectos de investigación y aborda el objeto de estudio desde una perspectiva múltiple.

Debido a las limitaciones de espacio, en este trabajo se presenta de manera resumida un panorama de los métodos y técnicas empleados para abordar el consumo de bienes culturales, que para los fines de los proyectos de investigación citados se ha definido como el conjunto de procesos socioculturales en que se realizan la apropiación, recepción y/o usos de los bienes producidos en el campo de la producción cultural.

\section{La encuesta}

Para cumplir con el objetivo de caracterizar el consumo de bienes culturales en sectores populares, se partió de un trabajo de Ranfla et al. (2001) en el que se establecieron cinco niveles de marginalidad para la zona urbana de Mexicali. Dicha propuesta, con base en el trabajo de Gino Germani (1973), señala que

puede definirse como marginalidad la falta de participación de individuos y grupos en aquellas esferas en las que de acuerdo con determinados criterios les correspondería participar. Por participación se entiende el ejercicio de roles o papeles concebidos de la manera más amplia: a) incluyen tanto un actuar, como un no actuar (producir o consumir), tanto obligaciones o deberes como

\footnotetext{
${ }^{1}$ Los tipos resultantes del análisis han sido descritos en L.M. Ortega, "Tipología del consumo de bienes culturales en Mexicali, B.C." , Frontera Norte, núm. 36, juliodiciembre 2006, pp. 53-85.
} 
derechos [...]; y b) [los roles] se ejercen en distintas instituciones y esferas de la vida individual y colectiva (así, se puede hablar de roles en la vida familiar, en el subsistema productivo, en el subsistema político, etc.) (Germani, 1973: 66-71, subrayado en la fuente).

En el trabajo de Ranfla et al. -y, por extensión, en el estudio sobre consumo de bienes culturales- se consideró el término marginalidad para denotar la falta de acceso a (o exclusión de) los beneficios de la inversión pública en infraestructura y distribución del ingreso, que se manifiestan en bajos índices de cobertura de los indicadores que fueron utilizados: porcentaje de casas sin agua entubada, sin drenaje, sin pisos de cemento, sin paredes de material durable (tabique o ladrillo), sin energía eléctrica, y de una sola habitación; porcentaje de población con un ingreso máximo de dos veces el salario mínimo, porcentaje de población analfabeta de 15 años o más, y porcentaje de población de 15 años o más sin educación media. Los resultados de ese estudio dieron como resultado la caracterización de cinco niveles de marginalidad, ${ }^{2}$ así como su distribución espacial en la mancha urbana de Mexicali, dividida en áreas geoestadísticas básicas (AGEB) según la definición del Instituto Nacional de Estadística, Geografía e Informática (INEGI, 2004).

El nivel 1 es el de menor marginalidad y corresponde a áreas geoestadísticas básicas (AGEB) con mayor equipamiento urbano, que en el estudio mencionado se asocia con mayores niveles de ingresos, y fue excluido del trabajo pues, de acuerdo con Alabarces (2004), refe-

\footnotetext{
${ }^{2}$ Cabe aclarar que la determinación de esos cinco niveles de marginalidad se llevó a cabo antes de que Conapo publicara los índices de marginación urbana, que consideran, además de los indicadores utilizados por Ranfla et al., el porcentaje de viviendas sin refrigerador, el porcentaje de viviendas con algún nivel de hacinamiento, el porcentaje de población que no son derechohabientes de los servicios de salud, el porcentaje de hijos fallecidos de las mujeres entre 15 y 49 años de edad, el porcentaje de población de 6 a 14 años que no asiste a la escuela, y el porcentaje de mujeres entre 12 y 17 años de edad que han tenido al menos un hijo nacido vivo. Por otra parte, los indicadores con los que se construyeron los índices de marginación urbana de Conapo no incluyen el porcentaje de viviendas sin pisos de cemento (Conapo, 2001).
} 
rirse a lo popular corresponde más a una tradición académica latinoamericana que a una categoría de análisis, ya que "si lo popular es diferencia o afirmación de una distinción conflictiva" (Alabarces, 2004: 26), esa diferencia remite a una situación de inferioridad (que el autor prefiere denominar como subalternidad), y que en el caso de este trabajo se establece por contraste con ese sector de población de menor nivel de marginalidad, que es el nivel 1.

Además, se eliminó también del estudio a las AGEB identificadas en el quinto nivel de marginalidad, pues en ellas se ubican algunas colonias que no cuentan con acceso a electricidad, de especial importancia para la recepción de los medios de comunicación electrónica, en particular la televisión, y que además implicaban riesgos para la seguridad de los encuestadores. Así, los sectores populares fueron ubicados en las AGEB con niveles 2 a 4 de marginalidad (90 en total), que de acuerdo con INEGI (2000) incluyen 99574 viviendas y representan el $72 \%$ de las viviendas de la ciudad de Mexicali, B.C.

A partir del tamaño de la población objeto de estudio, la muestra fue calculada con base en Scheaffer, Mendenhall y Ott (1987) con un nivel de confianza de $95 \%$. El resultado fue de 399 viviendas, cifra a la que se sumó un $10 \%$ debido a la posible no respuesta, con lo que se tuvo un total de 439 viviendas en una muestra proporcionada, esto es, una cuya composición guarda la misma estructura que la de los niveles de marginalidad en el total de las AGEB consideradas, que fueron numeradas para su selección aleatoria. Además, en los casos en que en una AGEB había más de una colonia, se numeraron las colonias para seleccionarlas aleatoriamente. Posteriormente, y usando como marco muestral los planos de las colonias, se numeraron las manzanas para hacer nuevamente una selección aleatoria de las mismas, y en cada manzana seleccionada se utilizó un número sistemático (4) para aplicar el cuestionario de la encuesta, empezando por la esquina noroeste de la manzana.

Los datos, que fueron procesados con el programa Statistical Package for the Social Sciences (SPSS), permitieron establecer una caracterización general del consumo de bienes culturales en sectores populares de Mexicali, tales como equipamiento presente en las viviendas, frecuencia de recepción de medios, salidas de fin de semana, etc. (véase 
Ortega y Ortega, 2005); y al hacer cruces de variables fue posible encontrar algunos rasgos significativos de acuerdo con el nivel de marginalidad, la edad de los miembros de las familias y, en ocasiones, hasta la colonia o el nivel educativo del informante. A partir de las tablas cruzadas, se hizo evidente que un análisis más detallado podría permitir entender mejor la estructura del fenómeno estudiado.

\section{El establecimiento de una tipología}

Los métodos de minería de datos se orientan hacia el análisis heurístico de información y su ventaja reside en que permiten manejar grandes volúmenes de información compleja. Entre dichos métodos, el análisis multivariado hace posible encontrar relaciones múltiples entre un buen número de variables, así como comparar y agrupar a los sujetos estudiados en función de similitudes en dichas relaciones, lo que sirve de base para el establecimiento de tipologías.

En el caso de este trabajo se utilizó el programa denominado Système Portable pour l'Analyse de Donées (SPAD), con el cual se llevó a cabo la caracterización de variables nominales y el análisis factorial (componentes principales, correspondencias múltiples, descripción de ejes factoriales), como pasos previos y necesarios para realizar los procesos de clasificación: partición de los individuos en clases y caracterización de las mismas.

Para ello, la base de datos en SPSS, que contiene 126 variables, fue reducida a una de 71, de entre las que se seleccionaron 68 variables categóricas y 3 ordinales, que fueron transformadas al formato del SPAD.

Dado que la determinación del número de clases por establecer es una prerrogativa del investigador, se hicieron diversos procesos $\mathrm{y}$, con base en el tamaño de las clases (número de viviendas en cada una) y en la distancia entre las mismas (expresadas en un diagrama o árbol de clases) se observó que la clasificación en cuatro clases era la que presentaba una estructura jerárquica más cercana a la piramidal, con lo que era posible encontrar diferencias marcadas entre aquéllas, además de que se evitaba tener algunas clases integradas por un muy reducido número de casos. 
Esta estructura, surgida de los propios datos de la encuesta, fue la base para la elaboración de una tipología del consumo de bienes culturales aplicable a los sectores populares de Mexicali tal como aquí se han definido, cuya caracterización se hizo a partir de las variables que resultaron más significativas; esto es, con valor test ${ }^{3}$ mayor o igual a 2.0 , lo que implica que sea significativo $(£ 0.05)$ y, por lo tanto, influyente en la definición del perfil de la clase (Bécue y Balls, 2005).

No obstante que los rasgos con los que se describe a cada clase están sustentados en las variables significativas, en su presentación como tipos de consumo de bienes culturales cabe destacar que, como dice Velasco (2001: 289), "el 'tipo' alude a una serie de rasgos recurrentes, generales y distintivos que no son propiedades atribuidas a un individuo sino al agregado del cual forma parte".

Asimismo, cabe distinguir el proceso de construcción de los tipos mediante procesos matemáticos, de aquel presentado por Weber (1973) como tipo "ideal", que corresponde a una construcción conceptual hecha por el investigador, hasta cierto punto intuitiva, síntesis de los rasgos esenciales ("genéticos") de un fenómeno estudiado, construcción contra la cual se compara la realidad empírica. La diferencia entre el tipo ideal weberiano y los que en este trabajo se obtuvieron está dada por el proceso mismo de construcción: para Weber, la construcción del tipo ideal se da antes de su contrastación empírica; en el caso de este trabajo, los datos empíricos son los que dan pie a la construcción de los tipos.

Sin embargo, para Weber los tipos ideales pueden entenderse como "conceptos abstractos de conexiones que, permaneciendo en el flujo

\footnotetext{
${ }^{3}$ De acuerdo con el glosario del SPAD, el valor test es un "Criterio estadístico que permite clasificar por orden de importancia los elementos característicos de un grupo de objetos" (CISIA, 1998: anexo A4). El valor test está asociado a la probabilidad de que la media de una variable en una clase determinada sea significativamente diferente a la media general de esa misma variable en el total de la muestra. Así, a mayor valor test más diferente es una clase respecto del promedio general y, por lo tanto, esa variable se puede asumir como característica de la clase. El valor test se interpreta como valor Z; es decir, cualquier valor test mayor o igual a 2 implica que la media de la clase es significativamente diferente de la media de toda la muestra.
} 
del acaecer, son representadas por nosotros como individuos históricos en los cuales se cumplen ciertos desarrollos" (Weber, 1973: 90, cursivas en el original), y en tal sentido, al igual que los tipos ideales de Weber, los tipos descritos a partir de los datos empíricos siguen siendo construidos, aunque más precisamente, son "extraídos" (McKinney, 1966); e "ideales" en el sentido de que permiten comprender la especificidad de un determinado fenómeno cultural, pues si bien a partir del procesamiento matemático se establecen correlaciones significativas entre variables que son comunes a un grupo de individuos, la expresión conceptual del tipo así obtenido corre a cargo del investigador, en un proceso de interpretación de lo que las correlaciones manifiestan; o dicho en palabras del propio Weber: "cuanto más se formen conceptualmente conexiones históricamente complicadas, en aquellos de sus elementos [de un fenómeno social] en los que estriba su específica significación cultural, tanto más el concepto -o el sistema conceptual -presentará el carácter del tipo ideal" (Weber, 1973: 90, cursivas en el original).

Para McKinney (1966: 216), "el tipo ayuda a manejar interrelaciones simultáneas y complicadas entre un número relativamente grande de variables, de manera preliminar, antes del desarrollo de la posibilidad operacional de manejarlas más rigurosamente respecto de un problema particular" (cursivas en el original). Gracias al desarrollo de sistemas de procesamiento de datos que apenas se avizoraba en los años sesenta, podría considerarse que la construcción de una tipología como la que se logró sería la concreción de aquella "posibilidad operacional". Una de las ventajas del programa utilizado es que, además de informar sobre las variables significativas de cada clase, muestra los sujetos que se ubican en cada una de ellas; en este caso, las viviendas según el número de folio del cuestionario aplicado durante la encuesta.

A partir de las cuatro clases que se obtuvieron con el análisis multivariado, se identificaron los tipos con letras y frases que aluden a los rasgos más sobresalientes: A) alto consumo, alto equipamiento, edades diversas; B) equipamiento medio, consumo medio, niños y adultos; C) televisión como equipamiento, consumo bajo, adultos; y D) muy bajo equipamiento, muy bajo consumo, adultos mayores (las 
características específicas de cada tipo han sido descritas en Ortega, 2006). Como se puede apreciar, los tipos A y D constituyen los extremos de un continuo, a la manera de los "tipos polares" de McKinney (1966), pero, nuevamente, construidos en momentos diferentes a los que plantea ese autor con base en Weber, pues la identificación de los polos y la ubicación de los tipos intermedios fue posterior a la recopilación de los datos y al establecimiento de las clases, y no previa, como en el método propuesto por McKinney (1966).

\section{La entrevista}

Los cuatro tipos de consumo de bienes culturales fueron un paso más para la comprensión del objeto de estudio, ya que hicieron posible dar cuenta de regularidades relacionadas con la composición de las familias, las edades de los miembros, las preferencias televisivas, el grado de equipamiento, el nivel educativo del informante, las salidas de esparcimiento y la asistencia a espacios de cultura legitimada (en el sentido de Bourdieu, 1984, 1993), entre otros aspectos. Por ejemplo, se hizo evidente, a través de la tipología, que las familias en donde hay niños tienen un consumo de bienes culturales más diversificado que aquellas sin menores, al tiempo que se descubrió una situación que en la encuesta no se había revelado: la exclusión que viven los adultos mayores, considerada como una manifestación de marginalidad cultural (Ortega, 2006), que se refiere a que, dentro de la tipología elaborada, las viviendas con un bajo o muy bajo consumo de bienes culturales se ubican en las AGEB con mayores niveles de marginalidad socioeconómica, a lo que se suma la exclusión en el acceso al equipamiento mediático sobre todo y, junto con ello, la restricción en el consumo de bienes culturales o al menos en las opciones para realizarlo. Ante esto cabe aclarar que si bien los medios de comunicación masiva no son los únicos espacios de circulación de bienes simbólicos, sí son -en la cultura contemporánea- los principales productores y difusores de aquéllos (Thompson, 1990), por lo que las restricciones en su acceso implican formas específicas de exclusión cultural. Esa situación de exclusión en el acceso a bienes simbólicos fue denominada marginalidad cultural a partir de que se considera a la marginalidad 
como "falta de participación de individuos y grupos en aquellas esferas en las que de acuerdo con determinados criterios les correspondería participar" (Germani, 1973: 66), que, en este caso, son criterios establecidos por las propias instituciones de cultura a través de los fines que promueven como propios: la extensión de la cultura y los servicios para enriquecer a la comunidad externa, en el caso de la Universidad Autónoma de Baja California (UABC, 2003); o “Generar condiciones para que la sociedad tenga acceso a bienes, servicios y actividades artístico-culturales como elementos esenciales del desarrollo humano integral", propósito del Instituto de Cultura de Baja California (Poder Ejecutivo de B. C., 2002: 17).

Como en todo proceso de investigación, los resultados dieron pie a nuevas preguntas, que esta vez requerían de otro tipo de abordaje metodológico. No se trataba ya de caracterizar, sino de entender cómo es que en los tipos identificados el consumo de bienes culturales llega a estructurarse del modo en que se presentaba, y para ese fin fue la entrevista la técnica seleccionada, debido a su capacidad para penetrar en el significado que para los sujetos entrevistados y sus familias tiene el consumo de bienes culturales, y a partir de ello poner de manifiesto la valoración que de él hacen y que da pie a las decisiones que se toman al respecto, así como la manera en que se hacen las elecciones ya identificadas por la encuesta.

De modo que fueron los tipos los que sirvieron de base tanto para seleccionar a las viviendas en las que se realizarían las entrevistas, como para establecer lo que se convertiría en las categorías de análisis de las mismas. Es con base en este procedimiento que se puede afirmar que hubo un muestreo cualitativo en el sentido de Denman y Haro (2000: 32), pues obedeció a la "inclusión de representantes de los diferentes estratos o situaciones sociales en que se expresa un fenómeno social", en donde los criterios de selección y exclusión estuvieron dados por la conformación de las clases con base en variables seleccionadas por el investigador que corresponden a la operacionalización del concepto eje -consumo de bienes culturales.

En cada clase reportada por el SPAD es posible identificar, además de las viviendas que la integran, aquellas que se ubican en los límites superiores e inferiores de cada clase; es decir, por ejemplo, en el caso 
de la clase que muestra mayor consumo, las viviendas del límite inferior resultan muy cercanas a las del límite superior de la clase de consumo medio, y las del límite inferior de esta clase se acercan a las del límite superior de la clase con bajo consumo, etc. De ahí que para la selección de las viviendas se optó por elegir dos de las que se ubicaban en los límites superiores de cada clase, y sólo en caso de no estar disponibles se recurrió a las que se ubicaban en la zona media, procurando excluir, en todo caso, a las de los límites inferiores.

Por otra parte, y con sustento en las características de los tipos, se establecieron premisas orientadoras que fueron la base para definir las categorías de análisis y las seis preguntas eje de las entrevistas.

Puesto que la situación de entrevista es ante todo una interacción, si bien provocada, se partió de los postulados de Goffmann (1981) para ubicar a la entrevista como una interacción dialógica (un par adyacente) que se desarrolla a partir de una serie de declaraciones y respuestas -si bien las declaraciones adquieren forma de interrogaciones- en la que se asume que existe un entendimiento mutuo que es más bien un acuerdo de trabajo "para fines prácticos" (Goffman, 1981: 10), entendimiento que puede no ser evidente para quien lee las transcripciones, por lo que una breve descripción del contexto de interacción y de los gestos del interlocutor son intercalados en las mismas, a fin de evitar el "pecado de descontextualización" (Goffmann, 1981: 32).

Ese entendimiento mutuo, que aparece como "natural" debido a que se comparte la misma lengua es, a decir de Verón (1998), una consecuencia de la eficacia de las leyes que rigen el funcionamiento de la lengua, ya que

[...] dentro de una comunidad dada, todos los miembros "normales" de ésta, confrontados con expresiones cualesquiera que forman parte de la lengua, las asociarán necesariamente y de manera automática con las mismas significaciones, ya sea en el nivel de las palabras o de las frases (Verón, 1998: 159).

Pero tal eficacia no es para dicho autor justificación del recurso a la "intuición" como criterio para la evaluación de los datos lingüísticos. 
Por ello, la entrevista es considerada en este trabajo como un proceso de producción de sentido, y con esto se asumen las dos hipótesis establecidas por Verón (1998: 125), a saber: a) que toda producción de sentido es necesariamente social; y b) que todo fenómeno social es, en una de sus dimensiones constitutivas, un proceso de producción de sentido. El producto de aquel proceso es, para Verón, un conjunto discursivo, el discurso, que tiene una manifestación material que en este caso es la transcripción de la entrevista y los señalamientos sobre las acciones de los entrevistados.

$\mathrm{Al}$ ser productos sociales, los discursos se hallan determinados tanto por las restricciones de su generación -condiciones de produccióncomo por las de su recepción -condiciones de reconocimiento-. "Generados bajo condiciones determinadas, que producen sus efectos bajo condiciones también determinadas, es entre estos dos conjuntos de condiciones que circulan los discursos sociales" (Verón, 1998: 127). De ahí, el autor afirma que analizar los discursos es describir las huellas de las condiciones productivas (de generación o de reconocimiento) en ellos, y niega la disputa acerca de la posibilidad de un análisis exclusivamente "externo" o "interno" de aquellos, ya que el objeto de análisis no está dentro ni fuera de los discursos, sino que son "sistemas de relaciones que todo producto significante mantiene con sus condiciones de generación por una parte, y con sus efectos por la otra" (Verón, 1998: 128).

Es precisamente ese sistema de relaciones al que se pretende aprehender con el análisis. No obstante, es menester tener en cuenta que para Verón un objeto significante admite lecturas múltiples, y que
[...] Sólo deviene legible en relación con criterios que se deben explicitar y que movilizan siempre, de una manera u otra, elementos que tienen que ver con las condiciones producti- vas del objeto significante analizado (sea en producción o en reconocimiento) (Verón, 1998: 128).

Por ello, en este trabajo los criterios utilizados -y explicitados- son los que se presentan como categorías de análisis y que se desprenden de las premisas orientadoras. Es desde dichos criterios -de reconoci- 
miento- que el discurso se ha hecho legible para el análisis del consumo de bienes culturales. Parafraseando a Verón (1998: 130), se podría entonces afirmar que el análisis de las entrevistas no es su interpretante, sino el signo de las mismas.

En resumen, la entrevista, como interacción social y proceso de producción de sentido, se objetiva en la transcripción que aparece como diálogo entre entrevistador y entrevistado. En dicho proceso, el sentido está dado por las relaciones del producto significante con sus condiciones de producción y de reconocimiento y las restricciones que establecen. Entre las condiciones de producción más relevantes habría que considerar que la misma situación de entrevista se presenta como una interacción "no espontánea", que forma parte de un proceso de investigación legitimado desde el campo académico y que goza de reconocimiento social (los "otros discursos" que menciona Verón), donde los actores de la interacción mantienen posiciones distintas y, por ello, poseen recursos diferenciados (Thompson, 1990): la entrevistadora tiene una posición privilegiada en el campo académico mientras que el entrevistado, si bien llega a ser incorporado en ese campo en tanto sujeto constituido en la situación de entrevista, tiene una posición subordinada. Así, el discurso llevará las huellas de estas condiciones.

En concordancia con lo anterior, Thompson (1990) afirma que las formas simbólicas -la entrevista como discurso es una de ellas- pueden portar las huellas de sus condiciones sociales de producción, y que están sujetas a reglas de codificación y de decodificación que no necesariamente son las mismas ni se aplican de igual forma por quien(es) produce(n) y por quien(es) recibe(n) dichas formas simbólicas.

Siguiendo a Verón (1998), se puede afirmar que, del mismo modo, las condiciones de reconocimiento, los "efectos" del discurso, se analizan a partir de una "gramática de reconocimiento" -operaciones de asignación de sentido-, establecida previamente (las categorías de análisis), y que se manifiesta como texto producido, como los resultados del análisis. De ese modo, y retomando lo expresado por Geertz (2001) respecto de la labor del etnógrafo, es posible decir que en el análisis de las entrevistas no sólo se "escribe", sino que a la vez se "inscribe" el discurso: 
El etnógrafo "inscribe" discursos sociales, los pone por escrito, los redacta. Al hacerlo, se aparta del hecho pasajero que existe sólo en el momento en que se da y pasa a una relación de ese hecho que existe en sus inscripciones y que puede volver a ser consultada (Geertz, 2001: 31)

Ese texto producido, a su vez, será el punto de partida de un reconocimiento por parte del lector, quien a su vez podrá analizarlo con base en sus condiciones de generación y de reconocimiento, para producir un nuevo texto, en la infinita red de la semiosis social en que se construye lo real.

\section{Premisas}

Para la definición del concepto consumo de bienes culturales, tal como se entiende en este trabajo, se recurrió a las aportaciones hechas por García Canclini (1993) en El consumo cultural en México, y las de Pierre Bourdieu (1993) en The Field of Cultural Production, con base en las cuales se elaboró la premisa correspondiente, que dice:

A partir de las aportaciones de García Canclini (1993) y de Bourdieu (1993), el consumo de bienes culturales se puede definir como el conjunto de procesos socioculturales en que se realizan la apropiación, recepción y/o usos de los bienes producidos en el campo de la producción cultural.

Partiendo de que el propósito que inspira el trabajo de investigación sobre consumo de bienes culturales es proveer de información que sea de utilidad no sólo para conocer de mejor manera las características con que se presenta aquél entre los sectores populares de Mexicali, sino -sobre todo- que sirva para fundamentar acciones congruentes con los propósitos que las propias instituciones de cultura establecen, y que se refieren a alcanzar a públicos más amplios, la premisa correspondiente dice:

Para el establecimiento de políticas y estrategias de promoción cultural pertinentes se hace necesario contar con infor- 
mación que dé cuenta de las especificidades regionales, por lo que una tipología del consumo de bienes culturales en la ciudad de Mexicali puede contribuir en tal sentido.

Con base en lo expuesto por Bourdieu (1984) respecto de cómo la disposición estética se adquiere por el contacto con las obras legítimas y por la forma de consumirlas, y cómo dicha disposición se manifiesta en prácticas específicas, sobre todo en la valoración de algunos productos y el rechazo de otros, se elaboró una premisa que toma en cuenta los antecedentes familiares y las experiencias pasadas en la familia, en tanto unidad de reproducción y aprendizaje del consumo de bienes culturales. Junto con ello -y si bien el nivel de marginalidad fue también una variable significativa en tres de los tipos (exceptuando el representado por una pareja de adultos mayores que viven solos), cuya importancia se refleja en el grado de equipamiento de las viviendas- se recurrió más que a considerar el capital económico, al concepto de origen social tal como lo aborda Borràs (1998), que se refiere sobre todo a la ubicación de los individuos en la producción, pues, a decir de este autor, los estudios sobre consumo tradicionalmente lo refieren al ingreso y "olvidan" que detrás de éste se encuentra el trabajo. Además, y ya que el nivel educativo del informante apareció como variable significativa, se estableció en la premisa correspondiente la importancia del capital educativo en la conformación de las características del consumo de bienes culturales, ya señalada y demostrada por Bourdieu (1984), de modo que la premisa quedó como sigue:

El capital educativo, el origen social y los antecedentes de los jefes de familia en materia de consumo de bienes culturales, son elementos articulados a las características de éste en las familias de los sectores populares de la ciudad de Mexicali, B. C., México.

Adicionalmente, y tomando en cuenta la vecindad de Mexicali respecto de Estados Unidos y la existencia de un mercado transfronterizo de bienes (González Aréchiga, 1985), se elaboró una premisa que establece que algunas características del consumo de bienes culturales pueden estar vinculadas a aquella condición: 
El consumo de bienes culturales en sectores populares de Mexicali, Baja California, México, se presenta con características asociadas a su ubicación geográfica de ciudad fronteriza con el sur de Estados Unidos de América.

Por otra parte, y dado que la composición de las familias resultó también significativa, se recurrió a algunos análisis que se han hecho desde la mercadotecnia y que señalan las estrategias utilizadas para la toma de decisiones respecto del consumo de productos y servicios, estrategias que son, a final de cuentas, manifestación de relaciones de poder entre los miembros de la unidad familiar (Kwai-Choi y Collins, 2000), por lo que se extrapoló dicha experiencia a la del consumo de bienes culturales, de manera que la premisa elaborada alude a las estrategias de resolución de conflictos que se ponen en práctica en las familias al momento de elegir los bienes culturales por consumir. También de los estudios sobre consumo de bienes y servicios se tomó el concepto de visiones del consumo (Phillips, Olson y Baumgartner, 1995), que se refiere a la elaboración de imágenes mentales sobre una situación de consumo, en este caso de bienes culturales, que permite dar cuenta del valor que los bienes involucrados tienen para el individuo. En este caso, partiendo del establecimiento que hace Thompson (1990) de las estrategias de evaluación simbólica de las formas simbólicas (entre las cuales se cuentan los bienes culturales), se enunció que en el consumo de bienes culturales se elaboran visiones de consumo que involucran estrategias de evaluación simbólica, en una premisa que dice:

En el consumo de bienes culturales se elaboran visiones de consumo (Phillips, Olson y Baumgartner, 1995) que involucran estrategias de evaluación simbólica (Thompson, 1990) y se aplican estrategias de resolución de conflictos en las que se hace uso de recursos de poder por parte de los miembros de las familias de los sectores populares.

\section{Epílogo}

La combinación de técnicas cuantitativas y cualitativas permite efectuar una triangulación efectiva y subsanar las insuficiencias de unas y 
otras; entre las más conocidas, las que se refieren a los niveles de generalización y profundidad, o las relativas a la descripción vs. la interpretación; de manera específica, el análisis multivariado aparece como una estrategia para el tratamiento de los factores rivales, tal como lo propone Denzin (2000).

Asimismo, este tipo de abordaje hace posible vincular la deducción propia de la perspectiva cuantitativa con la inducción característica de la cualitativa, en un trayecto que va de la teoría a la recopilación de datos vía encuesta para elaborar conclusiones, las cuales funcionan como datos que, sumados a los que aporta la entrevista, buscan enriquecer la teoría. Esquemáticamente, se podría representar del siguiente modo:

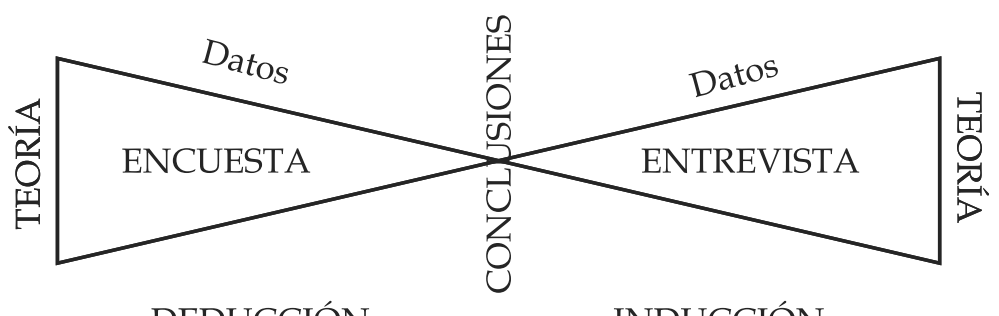

DEDUCCIÓN

INDUCCIÓN

Al momento de escribir este trabajo se está en las etapas de análisis de entrevistas y contraste de los resultados con la teoría. En esta fase es posible vislumbrar ya cómo algunas de las premisas se confirman y otras no, a la vez que surgen aspectos que sin haber sido considerados desde un principio se han convertido en temas de atención especial, como lo es el que se refiere a la construcción social de la realidad (Berger y Luckmann, 1968) y su manifestación en el discurso, debido sobre todo a la ausencia de referencias acerca de los bienes culturales legitimados por parte de los entrevistados, que no es necesariamente un problema de escasez de recursos lingüísticos, sino una expresión clara de lo que implica el "poder de nombrar" (Bourdieu, 2002). La apropiación de la dimensión referencial del mundo es condición de su apropiación material, dice Martín Serrano (1986); o en otras pala- 
bras, llegamos a ser dueños sólo de aquello que hemos podido nombrar. Por ello, y como conclusión adelantada, se puede establecer que dicha ausencia de referentes lingüísticos puede verse como una clara señal de la falta de contacto con la oferta de las instituciones y, por tanto, como exclusión de los beneficios del consumo de bienes culturales diferentes a los que se tiene acceso por la vía de los medios de comunicación masiva.

\section{Bibliografía}

Alabarces, Pablo (2004), “Cultura(s) [de las clases] populares, una vez más: la leyenda continúa. Nueve proposiciones en torno a lo popular", Potlatch. Cuaderno de Antropología y Semiótica, año I, número I, primavera de 2004. Disponible en línea http:/ / www. potlatch.com.ar/archivo_01.htm

Bécue, Mónica y Joan Balls (2005), Manual de introducción a los métodos factoriales y clasificación con SPAD, Servei d'Estadística Universitat Autònoma de Barcelona. Disponible en línea: www.uab.es/sestadistica/

Berger, P. L. y T. Luckmann (1968), La construcción social de la realidad, Argentina, Amorrortu.

Borràs, Vicent (1998), El consumo, un análisis sociológico. La estructura del consumo y los grupos sociales en la región metropolitana de Barcelona, Barcelona, Cedecs.

Bourdieu, P. (2002), Razones prácticas. Sobre la teoría de la acción, $3^{\text {a ed., }}$ Barcelona, Anagrama.

(1993), The Field of Cultural Production, Chicago, Columbia University Press. (1984), Distinction. A Social Critique of the Judgement of Taste, Cambridge, Harvard University Press.

Centre International de Statistique et d'Informatique Appliquées (CISIA) (1998), SPAD version 3. Manuel de prise en main 1998, Francia, CISIA-CERESTA.

Denman, C. A. y J. A. Haro (2000), “Introducción: Trayectoria y desvaríos de los métodos cualitativos en la investigación social", en C. A. Denman y J. A. Haro (comps.), Por los rincones. Antología de 
métodos cualitativos en la investigación social, Hermosillo, El Colegio de Sonora.

Denzin, N. K. (2000), "Un punto de vista interpretativo", en C. A. Denman, y J. A. Haro (comps.), Por los rincones. Antología de métodos cualitativos en la investigación social, Hermosillo, El Colegio de Sonora.

Douglas, M. y B. Isherwood (1979), El mundo de los bienes. Una antropología del consumo, México, Conaculta.

García Canclini, N. (1993), "El consumo cultural y su estudio en México: una propuesta teórica", en N. García Canclini (coord.), El consumo cultural en México, México, Conaculta.

Geertz, C. (2001), La interpretación de las culturas, Barcelona, Gedisa.

Germani, G. (1973), El concepto de marginalidad. Significado, raíces históricas y cuestiones teóricas, con particular referencia a la marginalidad urbana, Buenos Aires, Ediciones Nueva Visión, Colección Fichas. Goffman, E. (1981), Forms of Talk, Filadelfia, University of Pennsylvania Press.

González Aréchiga, B. (1985), “Aspectos estructurales del comercio fronterizo entre México y Estados Unidos", Estudios Fronterizos, núm. 6, México, UABC.

Instituto Nacional de Estadística, Geografía e Informática (INEGI) (2004). "Cartografía censal. Aspectos fundamentales". Disponible en: www.inegi.gob.mx/geografia/espanol/prodyserv/ cartocen / cartocen.cfm?c $=334$

(2000), XII Censo General de Población y Vivienda, Aguascalientes.

Kwai-Choi, C. y B.A. Collins (2000), "Familiy Decision Making and Coalition Patterns", European Journal of Marketing, vol. 34, núms. 9-10.

Martín Serrano, M. (1986), La producción social de comunicación, Madrid, Alianza Editorial.

McKinney, J. (1966), Constructive Typology and Social Theory, Nueva York, Appleton-Century-Crofts.

Ortega, G. (2006), “El consumo en las estrategias de reproducción en hogares pobres de la ciudad de Mexicali, B. C." , Tesis para obtener el grado de doctor en Ciencias Sociales Aplicadas, Tijuana, El Colegio de la Frontera Norte. 
Ortega, L. M. (2006), “Tipología del consumo de bienes culturales en Mexicali, B. C.", Frontera Norte, núm. 36.

Ortega, L. M. y G. Ortega (2005), Donde empieza la carne asada. Consumo de bienes culturales en sectores populares de Mexicali, Mexicali, UABC.

Phillips, D. M., J. C. Olson y H. Baumgartner (1995), “Consumption Visions in Consumer Decision Making", Advances in Consumer Research, núm. 22.

Poder Ejecutivo del Gobierno del Estado de Baja California (2002), Programa Estatal de Cultura, Mexicali, Instituto de Cultura de Baja California.

Ranfla, A., G. Ortega, G. Álvarez y D. Toudert (2001), “Estudio exploratorio de marginalidad en Baja California", Informe de investigación, Mexicali, Instituto de Investigaciones Sociales, UABC.

Scheaffer, R., W. Mendenhall y L. Ott (1987), Elementos de muestreo, México, Grupo Editorial Iberoamérica.

Sunkel, G. (coord.) (1999), El consumo cultural en América Latina, Bogotá, Convenio Andrés Bello.

Thompson, J. (1990), Ideology and Modern Culture, Stanford, Stanford University Press.

Universidad Autónoma de Baja California (2003), Plan de desarrollo institucional 2003-2006, Mexicali, UABC.

Velasco, M. L. (2001), “Un acercamiento al método tipológico en sociología", en M. L. Tarrés (coord.), Observar, escuchar y comprender. Sobre la tradición cualitativa en la investigación social, México, El Colegio de México, Flacso, Porrúa.

Verón, E. (1998), La semiosis social. Fragmentos de una teoría de la discursividad, Barcelona, Gedisa.

Fecha de recepción: abril de 2007

Fecha de aprobación: agosto de 2007 